\title{
Catalytic Condensation Methods for the Synthesis of Functionalized Diazoacetoacetates and Application to the Construction of Highly Substituted Cyclobutanones
}

\author{
Michael P. Doyle*, Kousik Kundu, and Albert E. Russell \\ Department of Chemistry and Biochemistry, University of Maryland, College Park, \\ Maryland 20742, USA.
}

\section{Supporting Information}

General. Reactions were performed in oven-dried $\left(140{ }^{\circ} \mathrm{C}\right)$ or flame-dried glassware under an atmosphere of dry $\mathrm{N}_{2}$. Dichloromethane was passed through a solvent column prior to use and was not distilled. Tetrahydrofuran and diethyl ether were distilled over sodium/benzophenone ketyl. Thin layer chromatography (TLC) was carried out using EM Science silica gel $60 \mathrm{~F}_{254}$ plates. The developed chromatogram was analyzed by UV lamp (254 nm), ethanolate phosphomolybdic acid, potassium permanganate $\left(\mathrm{KMnO}_{4}\right)$ or cerium ammonium molybdate (CAM). Liquid chromatography was performed using a forced flow (flash chromatography) of the indicated system on silica gel (230-400 mesh). Scandium(III), lanthanum(III), ytterbium(III), and copper(II) triflates were purchased from Aldrich and used as received. Methyl 3-tert-butyldimethylsilanyloxy-2-diazobut-3enoate was prepared by the method described by Davies. ${ }^{1}$

General Procedure for Mukaiyama Aldol Addition Reactions. To a dry 5-mL roundbottomed flask containing a stir bar and fitted with a septum was added anhydrous scandium(III) triflate $(14 \mathrm{mg}, 0.030 \mathrm{mmol})$ and $3 \mathrm{~mL}$ of anhydrous dichloromethane. Methyl 3-tert-butyldimethylsilanyloxy-2-diazobut-3-enoate (564 mg, $2.20 \mathrm{mmol}$ ) was then added dropwise over 5 min via a 1-mL syringe. Finally, benzaldehyde (152 $\mu \mathrm{L}$, $1.50 \mathrm{mmol}$ ) was added to the reaction mixture using a microsyringe. The reaction mixture was stirred at room temperature for $10 \mathrm{~h}$. (The reactions were found to be incomplete after $6 \mathrm{~h}$ for both benzaldehyde and p-anisaldehyde.) Over this time the solution changed color from orange to pale yellow. After the reaction was complete, the solution was passed through a silica gel plug to remove the catalyst with washings of 
dichloromethane. After removal of the solvent under reduced pressure, the product was isolated by flash chromatography.

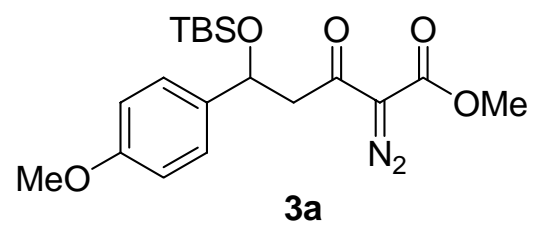

Methyl 5-(tert-Butyldimethylsilanoxy)-2-diazo-5-(p-anisyl)-3-oxopentanoate (3a). IR (neat): 2955, 2133, 1725, $1659 \mathrm{~cm}^{-1}$; ${ }^{1} \mathrm{H}$ NMR $\left(400 \mathrm{MHz}, \mathrm{CDCl}_{3}\right): \delta 7.26$ (d, $J=8.8 \mathrm{~Hz}$, 2H), 6.79 (d, $J=8.8 \mathrm{~Hz}, 2 \mathrm{H}$ ), 5.13 (dd, $J=8.8,4.0 \mathrm{~Hz}, 1 \mathrm{H}$ ), 3.77 (s, 3H), 3.73 (s, 3H), 3.46 (dd, $J=14.4,8.8 \mathrm{~Hz}, 1 \mathrm{H}$ ), 2.87 (dd, $J=14.4,4.0 \mathrm{~Hz}, 1 \mathrm{H}$ ), 0.77 (s, 9H), -0.07 (s, 3H), -0.24 (s, 3H); ${ }^{13} \mathrm{C}$ NMR (100 MHz, $\left.\mathrm{CDCl}_{3}\right): \delta 190.4,161.5,158.8,136.5,127.1$, 113.5, 71.6, 55.1, 52.1, 50.4, 25.6, 17.9, -4.8, -5.4; HRMS (FAB) for $\mathrm{C}_{19} \mathrm{H}_{28} \mathrm{~N}_{2} \mathrm{O}_{5} \mathrm{Si}$ [M+Li $]^{+}$calcd: 399.1928; found: 399.1909; TLC $\mathrm{R}_{f}=0.26$ (9:1 hexanes:ethyl acetate).

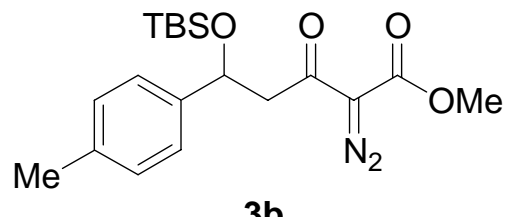

$3 b$

Methyl 5-(tert-Butyldimethylsilanoxy)-2-diazo-5-(p-tolyl)-3-oxopentanoate (3b). IR (neat): 2955, 2133, 1726, $1661 \mathrm{~cm}^{-1}$; ${ }^{1} \mathrm{H}$ NMR $\left(400 \mathrm{MHz}, \mathrm{CDCl}_{3}\right): \delta 7.27$ (d, $J=7.6 \mathrm{~Hz}$, 2H), 7.11 (d, $J=7.6 \mathrm{~Hz}, 2 \mathrm{H}$ ), 5.18 (dd, $J=9.2,4.0 \mathrm{~Hz}, 1 \mathrm{H}$ ), 3.83 (s, $3 \mathrm{H}$ ), 3.50 (dd, $J=$ 14.8, 9.2 Hz, 1H), 2.91 (dd, $J=14.8,4.0 \mathrm{~Hz}, 1 \mathrm{H}$ ), 2.33 (s, 3H), 0.82 (s, 9H), -0.03 (s, 3H), -0.20 (s, 3H); ${ }^{13} \mathrm{C}$ NMR (100 MHz, $\mathrm{CDCl}_{3}$ ): $\delta$ 190.4, 161.6, 141.4, 136.9, 128.8, 125.9, 71.9, 52.1, 50.5, 25.6, 21.1, 18.0, -4.8, -5.4; HRMS (EI) for $\mathrm{C}_{19} \mathrm{H}_{28} \mathrm{O}_{4} \mathrm{~N}_{2} \mathrm{Si}$ $[\mathrm{M}+\mathrm{Na}]^{+}$calcd: 399.1727; found: 399.1716; TLC $\mathrm{R}_{f}=0.27$ (9:1 hexanes:ethyl acetate). 


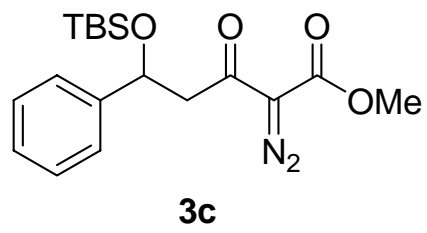

Methyl 5-(tert-Butyldimethylsilanoxy)-2-diazo-3-oxo-5-phenylpentanoate (3c). IR (neat): 2955, 2133, 1725, $1658 \mathrm{~cm}^{-1} ;{ }^{1} \mathrm{H}$ NMR (400 MHz, $\mathrm{CDCl}_{3}$ ): $\delta$ 7.37-7.22 (m, 5H), 5.20 (dd, $J=9.2,4.0 \mathrm{~Hz}, 1 \mathrm{H}), 3.81$ (s, 3H), 3.50 (dd, $J=14.8,9.2 \mathrm{~Hz}, 1 \mathrm{H}), 2.92$ (dd, $J=$ 14.8, 4.0 Hz, 1H), 0.81 (s, 9H), -0.03 (s, 3H), -0.21 (s, 3H); ${ }^{13} \mathrm{C}$ NMR (100 MHz, $\left.\mathrm{CDCl}_{3}\right): \delta 190.3,161.6,144.3,128.2,127.4,126.0,72.0,52.2,50.4,25.6,18.0,-4.8,-5.3$; HRMS (FAB) for $\mathrm{C}_{18} \mathrm{H}_{26} \mathrm{O}_{4} \mathrm{~N}_{2} \mathrm{Si}[\mathrm{M}+\mathrm{H}]^{+}$calcd: 363.1740; found: 363.1746; TLC $\mathrm{R}_{f}=$ 0.40 (9:1 hexanes:ethyl acetate).

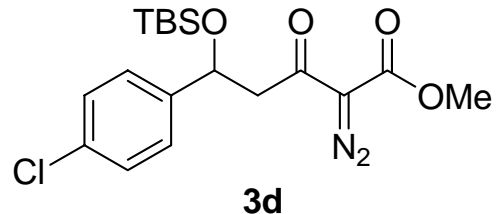

\section{Methyl 5-(tert-Butyldimethylsilanoxy)-5-(p-chlorophenyl)-2-diazo-3-oxopentanoate} (3d). IR (neat): 2928, 2135, 1718, $1653 \mathrm{~cm}^{-1} ;{ }^{1} \mathrm{H}$ NMR (400 MHz, $\mathrm{CDCl}_{3}$ ): $\delta 7.30$ (d, $J=$ 8.4 Hz, 2H), 7.24 (d, $J=8.8 \mathrm{~Hz}, 2 \mathrm{H}$ ), 5.17 (dd, $J=8.8,4.0 \mathrm{~Hz}, 1 \mathrm{H}), 3.79$ (s, 3H), 3.42 (dd, $J=14.8,8.8 \mathrm{~Hz}, 1 \mathrm{H}), 2.91$ (dd, $J=14.8,4.0 \mathrm{~Hz}, 1 \mathrm{H}), 0.79$ (s, 9H), -0.05 (s, 3H), 0.21 (s, 3H); ${ }^{13} \mathrm{C}$ NMR (100 MHz, $\left.\mathrm{CDCl}_{3}\right): \delta 190.0,161.5,143.0,133.0,128.4,127.4$, 71.2, 52.2, 50.3, 25.6, 18.0, -4.8, -5.3; HRMS (FAB) for $\mathrm{C}_{18} \mathrm{H}_{25} \mathrm{ClN}_{2} \mathrm{O}_{4} \mathrm{Si}[\mathrm{M}+\mathrm{Li}]^{+}$calcd: 403.1432; found: 403.1425; TLC $\mathrm{R}_{f}=0.40$ (9:1 hexanes:ethyl acetate).<smiles>COC(=O)C(=[W])CC(O[AsH3])c1ccc(C(F)(F)F)cc1</smiles> 
Methyl 5-(tert-Butyldimethylsilanoxy)-2-diazo-3-oxo-5-(p-trifloromethyl)pentanoate (3e). IR (neat): 2956, 2136, 1725, $1662 \mathrm{~cm}^{-1}$; ${ }^{1} \mathrm{H}$ NMR (400 MHz, $\mathrm{CDCl}_{3}$ ): $\delta 7.55$ (d, $J=$ $8.4 \mathrm{~Hz}, 2 \mathrm{H}$ ), 7.49 (d, $J=8.4 \mathrm{~Hz}, 2 \mathrm{H}$ ), 5.27 (ddd, $J=8.8,4.4 \mathrm{~Hz}, 1 \mathrm{H}$ ), 3.79 (s, 3H), 3.43 (dd, $J=15.2,8.8 \mathrm{~Hz}, 1 \mathrm{H}$ ), 2.95 (dd, $J=15.2,4.4 \mathrm{~Hz}, 1 \mathrm{H}$ ), 0.81 (s, 9H), -0.02 (s, 3H), 0.20 (s, 3H); ${ }^{13} \mathrm{C}$ NMR (100 MHz, $\mathrm{CDCl}_{3}$ ): $\delta$ 189.7, 161.5, 148.5, 126.2, 125.2, 125.1, 71.2, 52.1, 50.3, 25.5, 17.9, -4.9, -5.4; HRMS (FAB) for $\mathrm{C}_{19} \mathrm{H}_{26} \mathrm{~F}_{3} \mathrm{~N}_{2} \mathrm{O}_{4} \mathrm{Si}[\mathrm{M}+\mathrm{H}]^{+}$calcd: 431.1607; found: 431.1614; TLC $\mathrm{R}_{f}=0.30$ (7:1 hexanes:ethyl acetate).

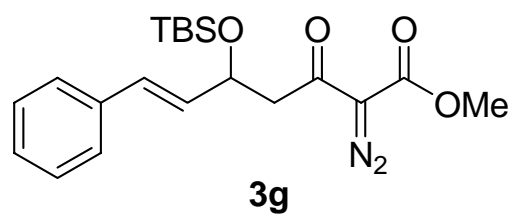

\section{Methyl (E)-5-(tert-Butyldimethylsilanoxy)-2-diazo-3-oxo-7-phenylhept-6-enoate (3g).} IR (neat): 2955, 2134, 1725, $1654 \mathrm{~cm}^{-1}$; ${ }^{1} \mathrm{H}$ NMR (400 MHz, $\mathrm{CDCl}_{3}$ ): $\delta$ 7.36-7.27 (comp, $5 \mathrm{H}$ ), 6.56 (d, $J=16 \mathrm{~Hz}, 1 \mathrm{H}$ ), 6.22 (dd, $J=16.0,6.8 \mathrm{~Hz}, 1 \mathrm{H}$ ), 4.84 (ddd, $J=7.6,6.8,5.2$ Hz, 1H), 3.81 (s, 3H), 3.32 (dd, $J=15.2,7.6 \mathrm{~Hz}, 1 \mathrm{H}$ ), 2.98 (dd, $J=15.2,5.2 \mathrm{~Hz}, 1 \mathrm{H}$ ), 0.87 (s, 9H), 0.05 (s, 3H), 0.04 (s, 3H); ${ }^{13} \mathrm{C}$ NMR (100 MHz, $\left.\mathrm{CDCl}_{3}\right): \delta$ 190.2, 161.6, 136.8, 132.0, 129.7, 128.5, 127.5, 126.5, 70.5, 52.2, 48.2, 25.7, 18.1, -4.3, -5.1; HRMS (ESI) for $\mathrm{C}_{20} \mathrm{H}_{28} \mathrm{~N}_{2} \mathrm{O}_{4} \mathrm{Si}[\mathrm{M}+\mathrm{Na}]^{+}$calcd: 411.1716; found 411.1707; TLC $\mathrm{R}_{f}=0.37$ (9:1 hexanes:ethyl acetate).

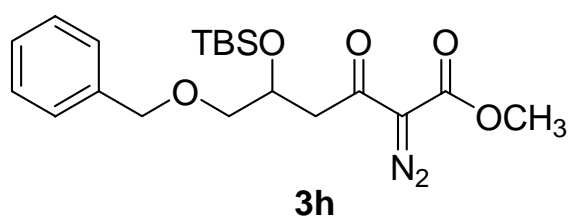

Methyl 6-(Benzyloxy)-5-tert-butyldimethylsilanoxy-2-diazo-3-oxohexanoate (3h). IR (neat): 2955, 2856, 2135, 1726, $1654 \mathrm{~cm}^{-1}$; ${ }^{1} \mathrm{H}$ NMR (400 MHz, $\left.\mathrm{CDCl}_{3}\right)$ : $\delta_{-} 7.31-7.29$ (comp, 5H), 4.5 (s, 2H), 4.42-4.41 (comp, 1H), 3.78 (s, 3H), 3.48 (dd, $J=9.6,5.2 \mathrm{~Hz}$, 1H), 3.40 (dd, $J=9.6,7.2 \mathrm{~Hz}, 1 \mathrm{H}$ ), 3.13 (dd, $J=15.2,7.2 \mathrm{~Hz}, 1 \mathrm{H}$ ), 3.05 (dd, $J=15.2$, $5.2 \mathrm{~Hz}, 1 \mathrm{H}), 0.83$ (s, 9H), 0.05 (s, $3 \mathrm{H}), 0.02$ (s, 3H); ${ }^{13} \mathrm{C}$ NMR (100 MHz, $\mathrm{CDCl}_{3}$ ): $\delta$ 
190.5, 161.6, 138.3, 128.3, 127.5, 127.5, 74.4, 73.2, 68.3, 52.1, 45.0, 25.7, 18.0, -4.6, 5.1; HRMS (FAB) for $\mathrm{C}_{20} \mathrm{H}_{30} \mathrm{~N}_{2} \mathrm{O}_{5} \mathrm{Si}[\mathrm{M}+\mathrm{H}]^{+}$calcd: 407.2002; found: 407.2002; TLC $\mathrm{R}_{f}$ $=0.33$ (9:1 hexanes:ethyl acetate).

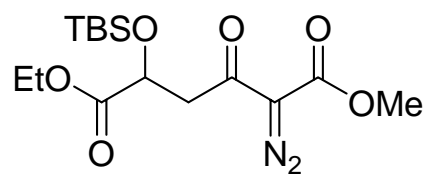

$3 \mathbf{i}$

Ethyl 1-Carbomethoxy-4-(tert-butyldimethylsilanoxy)-1-diazo-2-oxopentanoate (3i). IR (neat): 2930, 2135, 1729, $1660 \mathrm{~cm}^{-1}$; ${ }^{1} \mathrm{H}$ NMR (400 MHz, $\mathrm{CDCl}_{3}$ ): $\delta 4.69$ (t, $J=6.4$ Hz, 1H), 4.20-4.12 (m, 2H), 3.81 (s, 3H), 3.29 (d, $J=6.0 \mathrm{~Hz}, 2 \mathrm{H}$ ), 1.25 (t, $J=7.2 \mathrm{~Hz}$, 3H), 0.84 (s, 9H), 0.08 (s, 3H), 0.03 (s, 3H); ${ }^{13} \mathrm{C}$ NMR (100 MHz, $\mathrm{CDCl}_{3}$ ): $\delta$ 189.2, 172.6, 161.5, 68.6, 61.1, 52.2, 44.8, 25.6, 18.1, 14.1, -5.0, -5.5; HRMS (FAB) for $\mathrm{C}_{20} \mathrm{H}_{30} \mathrm{~N}_{2} \mathrm{O}_{5} \mathrm{Si}[\mathrm{M}+\mathrm{H}]^{+}$calcd: 359.1638; found: 359.1627; TLC $\mathrm{R}_{f}=0.19$ (9:1 hexanes:ethyl acetate).

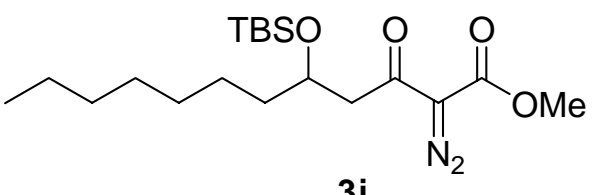

3j

Methyl 5-(tert-Butyldimethylsilanoxy)-2-diazo-3-oxododecanoate (3j). IR (neat): 2929, 2856, 2134, 1731, $1653 \mathrm{~cm}^{-1}$; ${ }^{1} \mathrm{H}$ NMR (400 MHz, $\mathrm{CDCl}_{3}$ ): $\delta$ 4.18-4.16 (m, 1H), 3.79 (s, 3H), 3.12 (dd, $J=15.2,7.6 \mathrm{~Hz}, 1 \mathrm{H}$ ), 2.80 (dd, $J=15.2,5.2 \mathrm{~Hz}, 1 \mathrm{H}$ ), 1.45-1.42 (m, 2H), 1.29-1.22 (m, 10H), 0.84 (t, $J=6.8 \mathrm{~Hz}, 3 \mathrm{H}), 0.80$ (s, 9H), -0.00 (s, 3H), -0.05 (s, 3H); ${ }^{13} \mathrm{C}$ NMR (100 MHz, $\left.\mathrm{CDCl}_{3}\right): \delta$ 191.2, 161.6, 69.2, 52.1, 47.1, 37.9, 31.7, 29.6, 29.2, 25.7, 24.9, 22.6, 17.9, 14.0, -4.7, -4.9; HRMS (FAB) for $\mathrm{C}_{19} \mathrm{H}_{36} \mathrm{~N}_{2} \mathrm{O}_{4} \mathrm{Si}[\mathrm{M}+\mathrm{H}]^{+}$ calcd: 385.2523; found: 385.2527; TLC $\mathrm{R}_{f}=0.46$ (9:1 hexanes:ethyl acetate). 


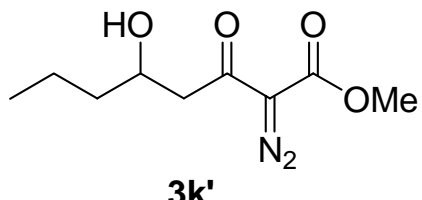

Methyl 2-Diazo-5-hydroxy-3-oxooctanoate (3k'). The TBDMS protecting group was removed by stirring the solution of $\mathbf{3 k}(0.10 \mathrm{~g}, 0.47 \mathrm{mmol})$ in methanol $(2 \mathrm{~mL})$ and silica gel (5 g) for 4 hrs at ambient temperature. IR (neat): 3498, 2959, 2137, 1723, 1657, 1649 $\mathrm{cm}^{-1}$; ${ }^{1} \mathrm{H}$ NMR (400 MHz, $\mathrm{CDCl}_{3}$ ): $\delta$ 4.07-4.01 (m, $1 \mathrm{H}$ ), 3.79 (s, 3H), 3.02 (dd, $J=17.2$, $2.8 \mathrm{~Hz}, 1 \mathrm{H}), 2.86$ (dd, $J=17.2,9.2 \mathrm{~Hz}, 1 \mathrm{H}), 1.51-1.30$ (m, 4H), $0.88(\mathrm{t}, 6.8 \mathrm{~Hz}, 3 \mathrm{H}) ;{ }^{13} \mathrm{C}$ NMR (100 MHz, $\left.\mathrm{CDCl}_{3}\right): \delta$ 192.9, 161.6, 67.8, 52.2, 46.8, 38.8, 18.6, 13.9; HRMS (FAB) for $\mathrm{C}_{9} \mathrm{H}_{14} \mathrm{~N}_{2} \mathrm{O}_{4}[\mathrm{M}+\mathrm{H}]^{+}$calcd: 215.1032; found 215.1025; TLC $\mathrm{R}_{f}=0.31$ (4:1 hexanes:ethyl acetate).

General Procedure for the Synthesis of Cyclobutanones. A solution of the Mukaiyama aldol adduct (1.0 mmol) in anhydrous $\mathrm{CH}_{2} \mathrm{Cl}_{2}(2 \mathrm{~mL})$ was added via syringe pump over 5 $\mathrm{h}$ to a refluxing solution of $\mathrm{Rh}_{2}(\mathrm{OAc})_{4}(4 \mathrm{mg}, 0.01 \mathrm{mmol})$ in anhydrous $\mathrm{CH}_{2} \mathrm{Cl}_{2}(2 \mathrm{~mL})$. The reaction mixture was then allowed to cool to room temperature and passed through a short silica gel plug to remove the catalyst with washings of dichloromethane. The solvent was removed under reduced pressure. The dominant product was isolated by flash column chromatography.

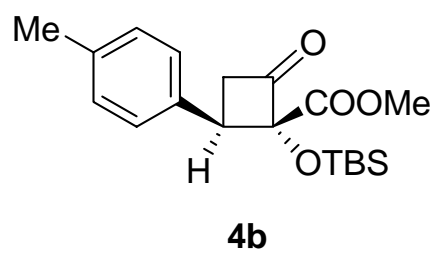

\section{Methyl 1-(tert-Butyldimethylsilanoxy)-2-oxo-4-(p-tolyl)cyclobutanecarboxylate (4b).} IR (neat): 2956, 2856, 1798, 1756, $1473 \mathrm{~cm}^{-1} ;{ }^{1} \mathrm{H}$ NMR (400 MHz, $\left.\mathrm{CDCl}_{3}\right): \delta$ 7.13-7.12 (comp, 4H), 3.74-3.60 (comp, 2H), 3.35 (s, 3H), 3.16 (dd, $J=16.4,9.6 \mathrm{~Hz}, 1 \mathrm{H}$ ), 0.93 (s, 9H), 0.17 (s, 3H), 0.14 (s, 3H); ${ }^{13} \mathrm{C}$ NMR (100 MHz, $\mathrm{CDCl}_{3}$ ): $\delta$ 201.7, 169.0, 136.8, 133.5, 129.0, 126.7, 51.6, 45.6, 44.3, 25.8, 21.1, 18.4, -3.6, -4.1; HRMS (EI) for 
$\mathrm{C}_{19} \mathrm{H}_{28} \mathrm{O}_{4} \mathrm{Si} \quad[\mathrm{M}+\mathrm{H}]^{+}$calcd: 348.1757; found: 348.1758; TLC $\mathrm{R}_{f}=0.34$ (19:1 hexanes:ethyl acetate).

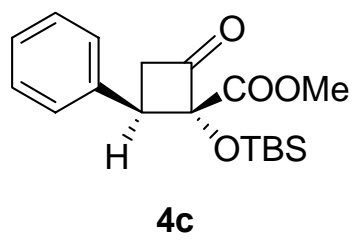

Methyl 1-(tert-Butyldimethylsilanoxy)-2-oxo-4-phenylcyclobutanecarboxylate (4c). IR (neat): 2954, 2855, 1797, 1754, $1462 \mathrm{~cm}^{-1} ;{ }^{1} \mathrm{H}$ NMR (400 MHz, $\mathrm{CDCl}_{3}$ ): $\delta$ 7.32-7.20 (comp, 5H), 3.76-3.60 (comp, 2H), 3.30 (s, 3H), 3.18 (dd, $J=16.4,9.6 \mathrm{~Hz}, 1 \mathrm{H}$ ), 0.94 (s, 9H), 0.16 (s, 3H), 0.12 (s, 3H); ${ }^{13} \mathrm{C}$ NMR (100 MHz, $\mathrm{CDCl}_{3}$ ): $\delta$ 201.5, 168.9, 136.7, 128.2, 127.2, 126.8, 97.4, 51.6, 45.8, 44.2, 29.6, 25.8, 18.3, -4.1, -5.4; HRMS (FAB) for $\mathrm{C}_{18} \mathrm{H}_{26} \mathrm{O}_{4} \mathrm{Si} \quad[\mathrm{M}+\mathrm{H}]^{+}$calcd: 334.1600; found: 334.1596; TLC $\mathrm{R}_{f}=0.34$ (19:1 hexanes:ethyl acetate).

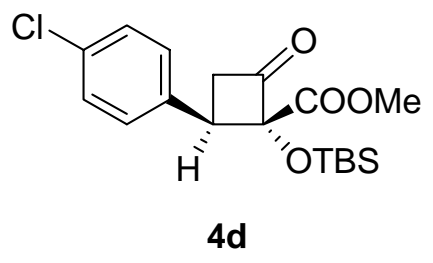

Methyl 1-(tert-Butlydimethylsilanoxy)-2-oxo-4-(p-chlorophenyl)cyclobutanecarboxylate (4d). IR (neat): 2954, 2857, 1798, 1756, $1493 \mathrm{~cm}^{-1}$; $\left.{ }^{1} \mathrm{H} \mathrm{NMR} \mathrm{(400} \mathrm{MHz,} \mathrm{CDCl}_{3}\right): \delta$ 7.29 (d, $J=8.4 \mathrm{~Hz}, 2 \mathrm{H}$ ), 7.14 (d, $J=8.4 \mathrm{~Hz}, 2 \mathrm{H}$ ), 3.71-3.56 (m, 1H), 3.35 (s, 3H), 3.17 (dd, $J=16.4,9.6 \mathrm{~Hz}, 1 \mathrm{H}), 0.91$ (s, 9H), 0.16 (s, 3H), 0.12 (s, 3H); ${ }^{13} \mathrm{C}$ NMR (100 MHz, $\left.\mathrm{CDCl}_{3}\right): \delta 200.9,168.8,135.2,133.1,128.5,128.2,97.4,51.7,45.4,44.2,29.7,25.8$, 18.3, -3.7, -4.1; HRMS (FAB) for $\mathrm{C}_{18} \mathrm{H}_{25} \mathrm{ClO}_{4} \mathrm{Si}[\mathrm{M}+\mathrm{H}]^{+}$calcd: 368.1211; found: 368.1209; TLC $\mathrm{R}_{f}=0.34$ (19:1 hexanes:ethyl acetate).

\section{Synthesis of 2-(tert-Butyldimethysilanoxy)-2-hydroxymethyl-3-(p-tolyl)cyclobutanol}

(6b). ${ }^{2}$ To an oven dried 10-mL round-bottomed flask containing magnetic stir bar and septum under nitrogen was added $1 \mathrm{~mL}$ anhydrous THF, followed by lithium aluminium 
hydride (1.5 mg, $1.0 \mathrm{mmol})$. The flask was then placed in an ice-water bath. $4 \mathbf{b}$ (201 $\mathrm{mg}, 0.570 \mathrm{mmol}$ ) was dissolved in $1 \mathrm{~mL}$ dry THF and was slowly added over $20 \mathrm{~min}$. The reaction mixture was then stirred at room temperature for $4 \mathrm{~h}$. After cooling the flask in an ice bath, $1 \mathrm{~mL}$ of water was added, and the product was extracted with THF (3 $\mathrm{x} 1 \mathrm{~mL}$ ) and then dried over anhydrous sodium sulphate. Solvent was removed under

reduced pressure. Product diol was isolated by flash column chromatography eluting with 3:1 hexanes:ethyl acetate to yield pure $\mathbf{6 b}$ (162 mg, 88\%, 95\% overall yield) in 12:1 diastereomeric ratio.

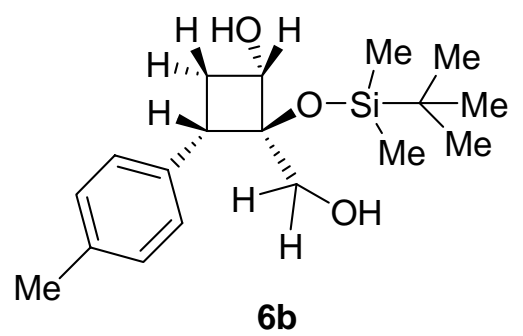

Major Isomer of 2-(tert-Butlydimethysilanoxy)-2-hydroxymethyl-3-(p-tolyl)cyclobutanol (6b). IR (neat): 3421, 2926, 2855, 1798, $1559 \mathrm{~cm}^{-1}$; ${ }^{1} \mathrm{H}$ NMR (400 MHz, $\mathrm{CDCl}_{3}$ ): $\delta 7.10$ (d, $J=8.0 \mathrm{~Hz}, 2 \mathrm{H}$ ), 7.06 (d, $J=8.0 \mathrm{~Hz}, 2 \mathrm{H}$ ), 4.29-4.25 (m, 1H), 3.76 (d, $J$ = $10.4 \mathrm{~Hz}, 1 \mathrm{H}$ ), 3.33 (d, $J=10.4 \mathrm{~Hz}, 1 \mathrm{H}), 3.17-3.12$ (m, 1H), 2.43 (ddd, $J=10.8,8.4,2.4$ Hz, 1H), 2.32 (s, 1H), 1.95-1.87 (m, 1H), 0.82 (s, 9H), -0.08 (s, 3H), -0.10 (s, 3H); ${ }^{13} \mathrm{C}$ NMR (100 MHz, $\left.\mathrm{CDCl}_{3}\right): \delta$ 136.3, 135.0, 129.3, 127.7, 80.9, 74.1, 63.6, 41.8, 26.2, 23.1, 18.3, -5.4, -5.5; HRMS (FAB) for $\mathrm{C}_{18} \mathrm{H}_{30} \mathrm{O}_{3} \mathrm{Si}[\mathrm{M}+\mathrm{Na}]^{+}$calcd: 345.1862; found: 345.1870 . 


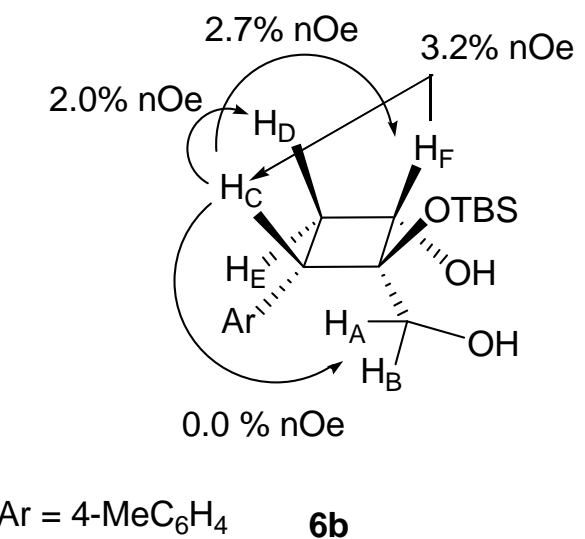

Determination of Relative Stereochemistry in $\mathbf{6 b}$. The relative stereochemistry of substituents in $\mathbf{6 b}$ was determined by nOe experiments with Gauss1.1000 shape pulse and gradient selection. Upon irradiation of the multiplet at $\delta 4.27 \mathrm{ppm}\left(\mathrm{H}_{\mathrm{F}}\right) 3.2 \%$ and $2.3 \%$ enhancements were observed for the multiplets at $\delta 3.15 \mathrm{ppm}\left(\mathrm{H}_{\mathrm{C}}\right)$ and at $\delta 2.43 \mathrm{ppm}$ $\left(\mathrm{H}_{\mathrm{D}}\right)$, respectively, and no enhancement was observed for the doublets at $\delta 3.76 \mathrm{ppm}\left(\mathrm{H}_{\mathrm{A}}\right)$ and $\delta 3.33 \mathrm{ppm}\left(\mathrm{H}_{\mathrm{B}}\right)$. Similarly upon irradiation of either of the doublets at $\delta 3.76 \mathrm{ppm}$ $\left(\mathrm{H}_{\mathrm{A}}\right)$ or $\delta 3.33 \mathrm{ppm}\left(\mathrm{H}_{\mathrm{B}}\right)$ no enhancement was observed for the multiplets at $\delta 3.15 \mathrm{ppm}$ $\left(\mathrm{H}_{\mathrm{C}}\right)$ and at $\delta 2.43 \mathrm{ppm}\left(\mathrm{H}_{\mathrm{D}}\right)$. But $2.7 \%$ and $2.0 \%$ signal enhancements were seen for multiplets at $\delta 4.27 \mathrm{ppm}\left(\mathrm{H}_{\mathrm{F}}\right)$ and $\delta 2.43 \mathrm{ppm}\left(\mathrm{H}_{\mathrm{D}}\right)$ upon irradiation of the multiplet at $\delta$ $3.15 \mathrm{ppm}\left(\mathrm{H}_{\mathrm{C}}\right)$. These observations could only be reconciled with the cis orientation of the aryl and hydroxymethyl group in the major diol product $\mathbf{6 b}$. Indirectly, these observations indicate $\mathbf{4 b}$ to be the major cyclobutanone product.

\section{General Procedure for Mannich Addition of 2 to Representative Benzylidene-} anilines. To a dry 5-mL round-bottomed flask containing a stir bar and fitted with a septum was added anhydrous scandium(III) triflate (14 mg, $0.030 \mathrm{mmol}$ ) and $2 \mathrm{~mL}$ of anhydrous dichloromethane. Methyl 3-(tert-butyldimethylsilanyloxy)-2-diazo-3butenoate 2 (564 mg, $2.20 \mathrm{mmol}$ ) was then added dropwise over $5 \mathrm{~min}$ via a 1-mL syringe. Finally, imine $(1.5 \mathrm{mmol})$ in $\mathrm{CH}_{2} \mathrm{Cl}_{2}(1 \mathrm{~mL})$ was added dropwise over $5 \mathrm{~min}$ to the reaction mixture. The reaction mixture was stirred at room temperature for $10 \mathrm{~h}$ (The reaction with 7a was found to be incomplete after $6 \mathrm{~h}$ ). Over this time the solution changed color from orange to bright yellow and was subsequently passed through a silica 
gel plug with washings of dichloromethane to remove the catalyst. Solvent was removed under reduced pressure. The TBDMS group was deprotected by stirring the solution of the Mannich adduct in methanol (5 mL) and silica gel $(5 \mathrm{gm})$ for $20 \mathrm{~min}$ at ambient temperature. Product was isolated by flash column chromatography eluting with 4:1 hexane:ethyl acetate.

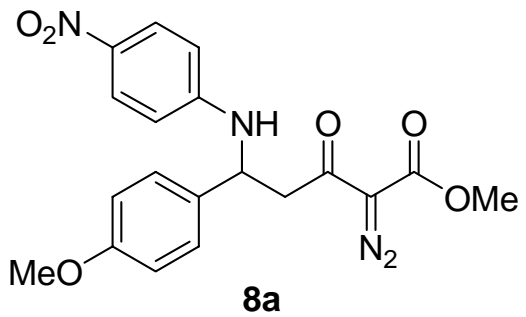

Methyl 2-Diazo-5-(4-methoxyphenyl)-5-(4-nitroanilino)-3-oxopentanoate (8a). IR (neat): 3375, 2953, 2148, 1721, $1643 \mathrm{~cm}^{-1} ;{ }^{1} \mathrm{H}$ NMR (400 MHz, $\mathrm{CDCl}_{3}$ ): $\delta 7.96$ (d, $J=$ $8.8 \mathrm{~Hz}, 2 \mathrm{H}), 7.28$ (d, $J=8.8 \mathrm{~Hz}, 2 \mathrm{H}), 6.84$ (d, $J=8.8 \mathrm{~Hz}, 2 \mathrm{H}), 6.44$ (d, $J=8.8 \mathrm{~Hz}, 2 \mathrm{H})$, 4.93-4.90 (m, 1H), 4.12 (bs, 1H), 3.85 (s, 3H), 3.75 (s, 3H), 3.37-3.35 (comp, $2 \mathrm{H})$ ) ${ }^{13} \mathrm{C}$ NMR (100 MHz, $\left.\mathrm{CDCl}_{3}\right): \delta$ 190.2, 161.9, 159.1, 152.1, 138.3, 132.7, 127.8, 127.2, 114.4, 112.1, 55.2, 54.2, 52.5, 46.9; HRMS (EI) for $\mathrm{C}_{19} \mathrm{H}_{18} \mathrm{~N}_{4} \mathrm{O}_{6}[\mathrm{M}+\mathrm{H}]^{+}$calcd: 398.1226; found: 398.1232; TLC $\mathrm{R}_{f}=0.20$ (4:1 hexanes:ethyl acetate).<smiles>COC(=O)C(=N)C(=O)CC(Nc1ccccc1)c1ccc(Cl)cc1</smiles>

Methyl 2-Diazo-5-(4-chlorophenyl)-5-anilino-3-oxo-pentanoate (8b). IR (neat): 3394, 2917, 2141, 1721, $1647 \mathrm{~cm}^{-1}$; ${ }^{1} \mathrm{H}$ NMR (400 MHz, $\mathrm{CDCl}_{3}$ ): $\delta 7.39$ (d, $\left.J=8.4 \mathrm{~Hz}, 2 \mathrm{H}\right)$, 7.29 (d, $J=8.4$ Hz, 2H), 7.10-6.47 (comp, 5H), 4.85 (dd, $J=9.6,4.0 \mathrm{~Hz}, 1 \mathrm{H}$ ), 4.69 (bs, 1H), 3.87 (s, 3H), 3.38 (dd, $J=14.4,4.0 \mathrm{~Hz}, 1 \mathrm{H}), 3.20$ (dd, $J=14.4,9.6 \mathrm{~Hz}, 1 \mathrm{H}$ ); ${ }^{13} \mathrm{C}$ NMR (100 MHz, $\left.\mathrm{CDCl}_{3}\right): \delta$ 190.8, 162.2, 146.9, 141.6, 133.4, 129.6, 129.3, 128.2, 118.3, 
114.0, 54.9, 52.9, 47.9; HRMS (EI) for $\mathrm{C}_{18} \mathrm{H}_{16} \mathrm{ClN}_{3} \mathrm{O}_{3}[\mathrm{M}+\mathrm{H}]^{+}$calcd: 357.0880; found: 357.0882; TLC $\mathrm{R}_{f}=0.29$ (4:1 hexanes:ethyl acetate).

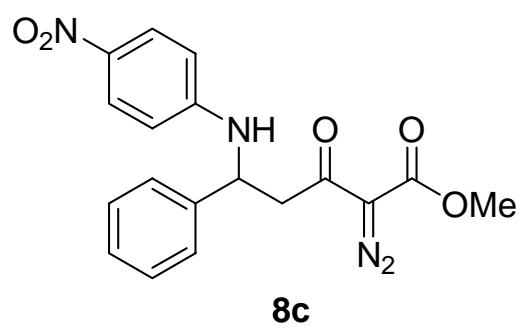

Methyl 2-Diazo-5-(4-nitrophenylamino)-3-oxo-5-phenylpentanoate (8c). IR (neat): 3353, 2134, 1716, $1652 \mathrm{~cm}^{-1} ;{ }^{1} \mathrm{H}$ NMR (400 MHz, $\left.\mathrm{CDCl}_{3}\right): \delta 7.97$ (d, $\left.J=8.8 \mathrm{~Hz}, 2 \mathrm{H}\right)$, 7.38-7.24 (comp, 5H), 6.44 (d, $J=8.8 \mathrm{~Hz}, 2 \mathrm{H}$ ), 5.67 (bs, 1H), 4.95-4.93 (m, 1H), 3.86 (s, 3H), 3.37-3.35 (m, 2H); ${ }^{13} \mathrm{C}$ NMR (100 MHz, $\left.\mathrm{CDCl}_{3}\right): \delta 190.1,161.9,152.0,140.7$, 138.4, 129.0, 127.9, 126.1, 126.0, 112.1, 54.8, 52.5, 46.8; HRMS (EI) for $\mathrm{C}_{18} \mathrm{H}_{16} \mathrm{~N}_{4} \mathrm{O}_{5}$ $[\mathrm{M}+\mathrm{H}]^{+}$calcd: 368.1121; found: 368.1112; TLC $\mathrm{R}_{f}=0.30$ (4:1 hexanes:ethyl acetate).

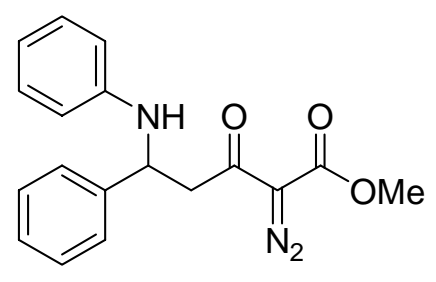

8d

Methyl 2-Diazo-5-anilino-3-oxo-5-phenylpentanoate (8d). IR (neat): 3365, 2132, 1720, $1641 \mathrm{~cm}^{-1} ;{ }^{1} \mathrm{H}$ NMR (400 MHz, $\mathrm{CDCl}_{3}$ ): $\delta$ 7.42-7.25 (comp, 5H), 7.05-6.49 (comp, 5H), 4.87 (dd, $J=9.6,4.4 \mathrm{~Hz}, 1 \mathrm{H}$ ), 4.67 (bs, $1 \mathrm{H}$ ), 3.82 (s, 3H), 3.37 (dd, 14.4, 4.4 Hz, 1H), 3.23 (dd, 14.4, $9.6 \mathrm{~Hz}, 1 \mathrm{H}) ;{ }^{13} \mathrm{C}$ NMR (100 MHz, $\mathrm{CDCl}_{3}$ ): $\delta$ 190.1, 162.3, 147.2, 143.0, 129.5, 129.1, 127.7, 126.7, 118.0, 114.0, 55.4, 52.8, 48.0; HRMS (EI) for $\mathrm{C}_{18} \mathrm{H}_{17} \mathrm{~N}_{3} \mathrm{O}_{3}$ $[\mathrm{M}+\mathrm{H}]^{+}$calcd: 323.3521; found: 323.3519; TLC $\mathrm{R}_{f}=0.33$ (4:1 hexanes: ethyl acetate). 


\section{References:}

(1) Davies, H. M. L.; Ahmed, G.; Churchill, M. R. J. Am. Chem. Soc. 1996, 118, 1077410780.

(2) Ramnauth, J.; Lee-Ruff, E. Can. J. Chem. 2001, 79, 114-120. 